\title{
Comment on "The effect of evaporation on the wicking of liquids into a metallic weave" by N. Fries, K. Odic, M. Conrath and M. Dreyer
}

\author{
D.A. Barry ${ }^{1, *}$, J.-Y. Parlange ${ }^{2}$, D.A. Lockington ${ }^{3}$, L. Wissmeier ${ }^{1}$ \\ ${ }^{1}$ Laboratoire de technologie écologique, Institut d'ingénierie de l'environnement, Station 2, Ecole polytechnique \\ fédérale de Lausanne, CH-1015 Lausanne, Switzerland. Ph. +41 (21) 693-5576, +41 (21) 693-5727; Fax. +41 (21) \\ 693-5670; E-mail addresses: andrew.barry@epfl.ch, laurin.wissmeier@epfl.ch \\ ${ }^{2}$ Department of Biological and Environmental Engineering, Cornell University, Ithaca, New York 14853-5701 USA. \\ Ph. +1 (607) 255-2476; Fax. +1 (607) 255-4080; E-mail address: jp58@cornell.edu \\ ${ }^{3}$ School of Engineering, University of Queensland, Brisbane, Queensland 4072 Australia. Ph. +61 (7) 3365-4054; \\ Fax.+61 (7) 3365-4599; Email address: d.lockington@mailbox.uq.edu.au
}

Submitted to: Journal of Colloid and Interface Science, 4 March 2009

Revised and Resubmitted 31 March 2009

\footnotetext{
* Author to whom all correspondence should be addressed.
} 
Fries et al. [1] reported careful experiments on capillary rise of a liquid in a weave both with and without evaporation. They used a model based on the assumption that the liquid wets the weave to saturation, i.e., the weave's saturation has only two states, completely saturated (all the pore space is filled with liquid) or completely unsaturated (all the pore space contains no liquid). They observed during liquid imbibition that, with evaporation, "there seems to be a trend that the mathematical model overestimates the height .... The average deviation is found to be in the range of $20 \% "$ ([1], pg. 127). Of various explanations for this discrepancy, they suggest that probably "the most influential is the assumption of a constant evaporation distribution all over the wet weave" and further suggested that their theory could explain the experimental data if "by some means the evaporation rate (might) be higher at the top of the weave". However, the authors present no mechanistic basis for this suggestion. Here, we propose an alternative to explain their observations.

We observe that the two-state, wet/dry model invoked by that authors, while a useful approximation for imbibition into a dry porous medium, is of only limited applicability for a draining or drying porous medium. For the case of imbibition into a dry medium with a limited range of pore sizes, a sharp saturation change at the wetting front is typical. For a medium with a saturated region that is allowed to drain or dry, there will typically be a gradual change in saturation throughout the medium. Case (6) in Fig. 10 shows the reduction in mass of a wetted weave due to evaporation. In their analysis of this case, Fries et al. ([1], pg. 124) deduced a constant mass drying rate per unit area of wetted weave. In other words, during evaporation the extent of the wetted weave did not change, only its saturation. Thus, the weave likely cannot be considered as being analogous with a capillary tube, which is either dry or saturated, rather it is a porous medium with a pore-size distribution, as suggested by Fig. 2. 
Our main point is that this fundamental assumption of two-state liquid content may not be justified. Their wetting model is based on the capillary bundle approach of Green and Ampt [2] or, more specifically, Washburn [3], which basically assumes a single pore size and could be a misleading description. Instead, we expect the weave to be saturated in the immersion zone and to be increasingly dry with height in the wicking zone. This is the case with or without a uniform evaporation flux but, as explained above, it is clear that with evaporation the drying weave experiences (up to) the complete range of saturations, from wet to dry.

The Green and Ampt model (Eq. 9 in [1]), which is the core of the evaporation/flow model developed by the authors, has been investigated physically for both downward and upward flow. The experiments in [1] all concern the latter case. In [4] it was shown that the Green and Ampt model incorporates a non-physical shape of the hydraulic conductivity as it varies with saturation. Because air entrapment - which is likely when a liquid moves in the direction of gravity due to the air buoyancy - changes the shape of the hydraulic conductivity especially near saturation, the Green and Ampt model can be considered as a reasonable approximation for vertically downward flow. However, for upward flow (as in the experiments in [1]), air entrapment is unlikely and the model's non-physical character could be a concern as the shape of the hydraulic conductivity function is not modified [4]. Indeed, based on their extensive laboratory experiments, Green and Ampt [2] found that their model was less applicable to vertically upward flow than to downward flow.

The authors defined $\phi$ as the porosity (volume of pore space divided by total volume), and obtained its value by measuring the slope in Fig. 11, which yields $\phi=0.24 \pm .03$ (called $\theta$ in Table 7). If the pore space is completely full with liquid, then the saturation is identical to the porosity. If the liquid only partially fills the available pore space, then the saturation will be lower 
than the porosity. For a medium containing no liquid the saturation is zero. Since for their experiments with evaporation they estimate the maximum height from mass measurements, taking $\phi$ $=0.24$ as if there was no evaporation, the actual $\phi$ should be lower and as a result we suspect that the actual height is greater than given in Fig. 13 for instance.

Based on the changing saturation within the weave, we now give a crude quantitative estimate of the $20 \%$ discrepancy noted by the authors following application of their two-state theory. The non-constant $\phi$ in the profile is largely due to the capillary diffusion of liquid in the weave. The capillary diffusion coefficient in a porous medium can be approximated by a power law, $\sim \phi^{n}$, with $4 \leq n \leq 8$ [5] (if $n \rightarrow \infty, \phi^{n}$ approaches a delta function in which case $\phi$ becomes uniform and, for imbibition, the two-state saturation model assumed by the authors ensues). The parameter $n$ is an empirical, medium-dependent constant. Without evaporation the liquid content $\phi$ is a function of position $x$ given by Eq. (6) in [6] as $x / x_{F}=1-\left(\phi / \phi_{0}\right)^{n}$, where $x_{F}$ is the position of the wetting front and $\phi_{0}$ is the value of $\phi$ at saturation. Then the average liquid content is given by $\left(x_{F} \phi_{0}\right)^{-1} \int_{0}^{\phi_{0}} x d \phi=n /(n+1)$. With evaporation the steady state profile is given by Eq. (12) in [7] as, $x / x_{F}=1-\left(\phi / \phi_{0}\right)^{n / 2}$, then the average liquid content is $n /(n+2)$. Thus evaporation reduces the average liquid content in the profile by $(n+1) /(n+2)$, or between approximately 10 and $20 \%$ for the range of possible values of $n$ given above. These estimates ignore the effect of gravity. Note also that Lockington et al. [7] considered the case when evaporation is proportional to the liquid content, whereas the authors showed that evaporation in their experiments is largely independent of liquid content. Then, the reduction for $\phi$ should even be greater than ( $n$ $+1) /(n+2)$. In conclusion, the actual heights with evaporation should be at least 10 to $20 \%$ high- 
er than the values they give (which are based on mass data), thereby reducing the discrepancy with the predicted values. 


\section{References}

[1] N. Fries, K. Odic, M. Conrath, M. Dreyer, J. Colloid Interface Sci. 321 (2008) 118-129.

[2] W.H. Green, G.A. Ampt, J. Agri. Sci. 4 (1911) 1-24.

[3] E.W. Washburn, Phys. Rev. 17 (1921) 273-283.

[4] D.A. Barry, J.-Y. Parlange, G.C. Sander, M. Sivaplan, J. Hydrol. 142 (1993) 29-46.

[5] R.H. Brooks, A.J. Corey, 1964. Hydraulic properties of porous media. Hydrol. Paper 3 (1964) Colo. State Univ., Fort Collins, Colorado, USA.

[6] W. Brutsaert, Water Resour. Res. 4 (1968), 785-789.

[7] D.A. Lockington, J.-Y. Parlange, M. Lenkopane, Transp. Porous Media 68 (2007) 29-36. 